\title{
ANÁLISIS ARCHIVÍSTICO DE LOS PROYECTOS DE CONSTRUCCIÓN DE LAS CASAS CUARTEL DURANTE EL FRANQUISMO: EL EJEMPLO DE LA PROVINCIA DE BADAJOZ
} (1943-1971)

\author{
Isabel Ma Sanz Caballero \\ Universidad de Extremadura \\ isancab@unex.es \\ Agustín Vivas Moreno \\ Universidad de Extremadura \\ aguvivas@unex.es
}

RESUMEN: El presente trabajo aborda el estudio de la documentación de los proyectos de construcción de casas cuartel que se llevaron a cabo en Extremadura tras la Guerra Civil Española (1936-1939). Se realiza, por tanto, un estudio sobre el contexto histórico y la legislación bajo la que fueron concebidos estos proyectos de construcción. Además, se realiza un análisis archivístico de dos de los expedientes que se encuentran conservados en el Archivo del Servicio Territorial de Badajoz de la Consejería de Movilidad, Transporte y Vivienda de la Junta de Extremadura. El objetivo del trabajo, en consecuencia, es dar a conocer la importancia de la documentación de los proyectos de construcción de casas cuartel en Extremadura, generada durante la Dictadura Franquista. Seguimos para ello una metodología histórica basada en tres fases: análisis funcional e institucional, análisis documental y análisis histórico. El resultado es identificar y analizar parte de la documentación de los proyectos constructivos de casas cuartel de primera y última época Franquista.

Palabras clave: Casas cuartel, Guardia Civil, proyectos de construcción, Franquismo, Regiones Devastadas. 


\title{
ARCHIVAL ANALYSIS OF THE CONSTRUCTION PROJECTS OF THE BARRACKS DURING THE FRANCO DICTATORSHIP: THE EXAMPLE OF BADAJOZ PROVINCE (1943-1971)
}

\begin{abstract}
This paper deals with the study of the documentation of construction projects of Guard Barracks Houses that took place in Extremadura after the Civil War Española (1936-1939). Therefore, a study on the historical context and the legislation under which these construction projects were conceived is carried out. In addition, an archival analysis of two of the files that are kept in the Archive of the Badajoz Territorial Service of the Ministry of Mobility, Transportation and Housing of the Junta de Extremadura is carried out. Through this analysis, we will be able to verify the characteristics of the documentation we are facing, as well as its historical purpose. The objective of the present work is to make known the importance of the documentation of the construction projects of barracks houses in Extremadura, generated during the Franco Dictatorship. The methodology has been determined by a series of phases such as the collection and study of the projects of Guard Barracks House in Extremadura during the Franco Dictatorship, preserved in the Archive of the Territorial Service of Badajoz, and the analysis of two dossier of the construction projects of Guard Barracks House of the first and last Franco Dictatorship period. The result of this analysis will reveal the differences in the documentation of the construction projects of Guard Barracks House of the first and last Franco Dictatorship period.
\end{abstract}

Keywords: Barracks, Civil Guard, construction projects, Franco Dictatorship, Devastated Regions.

Recibido: 27 de marzo de 2020

Aceptado: 23 de junio de 2020

\section{Introducción. La politica de viviendas en el Franquismo: planificación y periodización}

En este punto, cabe comentar brevemente que la base de partida de nuestro estudio vendrá determinada naturalmente por las huellas que han dejado otros autores en materia de urbanismo y obras públicas y sus múltiples y fragmentarias perspectivas. El amplio contexto de la temática obliga a una selección de obras, autores y corrientes.

Desde un punto de vista global, para la historia del urbanismo han sido fundamentales, entre otras, las obras de Capel (1975) -al tratar la vinculación en España entre el capitalismo y la morfología urbana-, el excelente manual de Chueca (1970) para la historia del urbanismo, todavía en uso a pesar de los años, y las reflexiones de Terán (1978) acerca de los planeamientos urbanos en España. 
Son abundantes los autores que abordan las políticas de vivienda. Por orden cronológico, por citar algunas, destaca la obra de Esteban (1999) y sus análisis sobre la vivienda social española de los años 50, la de Beltrán (2002) que examina desde un punto de vista genérico la política de vivienda en la España franquista y postfranquista, o la de Blasco (2002) que realiza una interesante perspectiva histórica sobre las diferentes políticas de vivienda en España.

Desde un punto de vista más institucional, son importantes las obras de Pérez del Hoyo y Gutiérrez (2013) que analiza las primeras políticas de vivienda en España y su influencia en la evolución de la tipología residencial, la de Sánchez (2014) que estudia el Instituto de Reformas Sociales, la de Ruiz (2015) sobre los derechos de los trabajadores en el franquismo, o la reciente de López (2018) sobre el chabolismo vertical, los movimientos migratorios y la política de vivienda franquista desde 1955 y hasta 1975.

Para ofrecer una base de los organismos surgidos tras la guerra civil en España, son de obligada consulta: para la Organización Sindical Española (OSE) y sus instituciones vinculadas la obra de Leal (1955); sobre el Instituto Nacional de Colonización, la de Jubert (1974); para la Obra Sindical del Hogar las de Gutiérrez-Mozo (2015) y Alquézar (2016); sobre la Obra Sindical de la Vivienda, la de Alagón y Vázquez (2015) sobre las Escuelas de "sabor agrario" en los pueblos creados por el Instituto Nacional de Colonización; o la de Centellas, et al. (2010) sobre los pueblos de colonización de la administración franquista. Por su parte, para el estudio del Servicio Nacional de Regiones Devastadas sirven de referente la obra clásica de López (1995) y su estudio para Aragón aunque de gran validez para toda España, o la de Ruesga (2016) que realiza un análisis entre lo estético y lo ideológico.

Con respecto a las casas cuartel de la Guardia Civil, es fundamental el estudio de Pinzón (2014) y su excelente análisis sobre las casas-cuartel de la Guardia Civil durante la II República y el franquismo, y más recientemente el que hace el mismo autor junto a Loren (2017) y su examen acerca de la casa cuartel de la Guardia Civil como vivienda colectiva regenerable. Al margen, conviene hacer mención para nuestros intereses, la tesis doctoral del citado Daniel Pinzón Ayala leída en la Universidad de Sevilla, titulada: "Una arquitectura para trabajar y vivir en colectividad: la casa-cuartel de la Guardia Civil" (2016)

Respecto a los contenidos archivísticos, no hay apenas obras que analicen de forma específica la temática en cuestión. Sí encontramos algunas referencias específicas en abundantes manuales, formando parte de contenidos globales, Asimismo, naturalmente son adaptables los contenidos sobre gestión documental, organización de archivos, descripción y normalización u obras sobre contenidos y tratamiento de la documentación autonómica. Lógicamente no sistematizaremos aquí las líneas historiográficas en este orden. Sí que conviene citar algunos autores que han resultado ser sustanciales para nuestros contenidos. Es el caso, por ejemplo, de Olga Gallego (1978) que, hace casi cincuenta años, analizó la documentación de las delegaciones provinciales del Ministerio de 
la Vivienda, obra que sigue siendo de gran valía. En este caso, como en tantos otros, las reticencias a las obras antiguas $-y$ el merecimiento por sistema de las más recientes- merecen el calificativo de absurdas. Del mismo modo, destaca la guía que realiza Fuente (1983) del Archivo General del Ministerio de Obras Públicas y Urbanismo, o la de Rojas y de Andrés (2015) sobre la historia institucional y documental del Ministerio del Interior. Sí que hay diferentes estudios de carácter provincial. Destaca el caso de Valladolid con obras de Candau (2005), Castro (2006) o Laso (2013) con diferentes estudios de la política de viviendas y los documentos de arquitectura y urbanismo en el Archivo Histórico provincial de Valladolid. No obstante, a pesar de todas estas referencias, encontramos un importante desierto historiográfico para Extremadura que intentamos ponerle solución con la presente obra.

\subsection{Periodización}

Para analizar la construcción de casas cuartel durante el franquismo debemos primeramente, siquiera de forma escueta, realizar un recorrido por la política de vivienda llevada a cabo en esta época. A continuación, se recoge de forma eficiente su planificación y periodización'.

Existen durante esta época dos etapas dirigidas a la política de vivienda:

- La primera, en el período 1939 a 1954, dirigida a la reconstrucción de viviendas tras la Guerra Civil Española.

- Y la segunda, en el período 1954 a 1976, que se caracteriza por la iniciativa privada en la construcción de viviendas protegidas.

Podemos denominar la primera etapa como "período de reconstrucción" (1939-1954), tras la Guerra Civil Española. En este primer período, surge por primera vez la definición legal de vivienda protegida con la Ley de 19 de abril de 1939, que incluyó la vivienda social -vivienda mínima-y las viviendas para militares o funcionarios. No fue hasta 1954 cuando se redactó una nueva legislación que refundía las categorías anteriores y generaba un nuevo tipo de viviendas pensando en resolver el problema de la vivienda más modesta ( $v i$ viendas de renta mínima y reducida), a la par que se creaba un Plan Nacional de la Vivienda².

La segunda etapa o "de despliegue" (1954-1976), por su parte, se caracterizó por los tres regímenes de viviendas diferentes que se reflejan a continuación:

1. Blasco Torrejón, B., Política de vivienda en España: un análisis global (Tesis Doctoral). Madrid 2002.

2. López Díaz, J., "Vivienda social y falange: ideario y construcciones en la década de los 40". Scripta Nova. Revista electrónica de geografía y ciencias sociales vol. VII, 146 (024) (2003), http://www.ub.edu/geocrit/sn/sn-146(024).htm [consultado 18/11/2019]. 
1. Régimen de "Viviendas de tipo Social" (Ley de 14-5-1954).

2. Régimen de "Viviendas de Renta Limitada" (Ley de 15-7-1954).

3. Régimen de "Viviendas Subvencionadas" (Ley de 13-11-1957).

\subsection{Régimen de vivienda de renta limitada: las casa cuartel}

De acuerdo con el objetivo de esta investigación, nos detendremos en el Régimen de Viviendas de Renta Limitada, por ser éste el tipo de régimen en el que va a edificar la tipología de viviendas en las que vamos a centrar nuestro estudio: las casas cuartel.

Los proyectos de construcción de las casas cuartel fueron publicados en el Boletín Oficial bajo la siguiente disposición "Conforme a lo dispuesto en la Ley, de quince de julio de mil novecientos cincuenta y cuatro, que establece el régimen de "viviendas de renta limitada", hecho extensivo a la Guardia Civil por Orden de la Presidencia del Gobierno se autoriza al Ministerio de la Gobernación para concertar con el Instituto Nacional de la Vivienda la operación oportuna para la construcción de un edificio destinado a casa-cuartel de la Guardia Civil"

En este orden, el Régimen de Viviendas de Renta Limitada se caracteriza por diferentes categorías:

a) Tipos de vivienda: No existía un concepto claro de la vivienda objetivo. Se establecieron tres categorías en función de los metros cuadrados útiles:

- Grupo III, no inferior a 42 metros cuadrados construidos.

- Grupo II, no superior a 125 metros cuadrados construidos.

- Grupo 1, no superior a 200 metros cuadrados construidos.

b) Destinatarios: Podían cederse en alquiler -por renta o incluso gratuitamente- o mediante compra-venta al contado o a plazos.

c) Medidas de Fomento: Se dirigían a la "piedra", es decir al promotor; se cuidaba la oferta, con lo que indirectamente se atendía a la demanda.

Las casas cuartel, también denominados cuartelillos, eran las instalaciones donde el personal de la Guardia Civil allí destinado (cuerpo que tomó gran importancia durante la Dictadura Franquista) desarrollaba tanto su labor profesional como su vida privada, normalmente junto a sus familias.

\section{Método}

Para la consecución de los objetivos que proponemos, seguimos la metodología histórica, basada en tres fases:

1. El análisis institucional: el objetivo es el análisis de las instituciones vinculadas para la comprensión de su estructura orgánica y funcional; 
2. El análisis documental, en dos etapas:

a. Por un lado, el análisis de la génesis documental y los procedimientos administrativos Ilevados a cabo para la construcción de casas cuartel, tomando como ejemplo la provincia de Badajoz;

b. Por otro, la clasificación documental y la identificación de las series relacionadas, estructurándolas en un Cuadro de Clasificación que se comporta como instrumento descriptivo.

3. Por último, el análisis histórico, dejando entrever las investigaciones históricas factibles que pueden ser base de estudio a través de la documentación identificada y descrita.

TABLA 1. Recursos metodológicos.

\begin{tabular}{|l|l|l|}
\hline \multicolumn{2}{|c|}{ METODOLOGÍA HISTÓRICA } \\
\hline Análisis institucional & $\begin{array}{l}\text { Examen institucional/ } \\
\text { funcional }\end{array}$ & $\begin{array}{l}\text { Comprensión de la estructura } \\
\text { orgánica y funciona }\end{array}$ \\
\hline $\begin{array}{l}\text { Análisis documental- } \\
\text { archivística }\end{array}$ & $\begin{array}{l}\text { Examen de la génesis } \\
\text { documental }\end{array}$ & Cuadro de clasificación \\
\cline { 2 - 3 } Identificación documental & $\begin{array}{l}\text { Identificación temáticas } \\
\text { históricas }\end{array}$ \\
\hline Análisis histórico & Análisis Histórico & \\
\hline SISTEMA DE INFORMACIÓN HISTÓRICO (SIH)
\end{tabular}

En definitiva, análisis institucional, documental e histórico como base de un Sistema de Información Histórica para el análisis de la construcción de las casas cuartel en el franquismo.

\section{Organismos relacionados con la construcción de las casas cuartel en el Franquismo. El Servicio Nacional de Regiones Devastadas}

En relación a lo expuesto, para el preceptivo análisis institucional, se hace necesario, en primer lugar, realizar un recorrido por los organismos relacionados con las políticas de vivienda en el franquismo. Sólo así, podremos analizar posteriormente uno de ellos: las casas cuartel de la Guardia Civil.

\subsection{El Servicio Nacional de Regiones Devastadas}

El Servicio Nacional de Regiones Devastadas y Reparaciones (SNRDR) fue un organismo creado durante la dictadura de Franco en España. Este organismo se creó en enero de 1938 con la finalidad de dirigir e inspeccionar los pro- 
yectos de reconstrucción, tanto de viviendas y monumentos artísticos, como de infraestructuras, dañadas por la guerra civil en el territorio denominado "nacional".

Finalizada la contienda, en agosto de 1939 pasó a denominarse Dirección General de Regiones Devastadas y Reparaciones (DGRDR) y a depender del Ministerio de la Gobernación.

Este organismo, junto con el de la Dirección General de Arquitectura (DGA), sería el encargado de la reconstrucción del país, en especial de aquellas regiones y territorios que habían resultado significativamente dañados por la contienda. Muchas reconstrucciones de iglesias, monumentos y edificios públicos se deben a aquella época. En septiembre de 1939, se decide que aquellas regiones con una destrucción superior al 75\% quedaban bajo su tutela de forma espacial.

Asimismo, los objetivos de la Dirección General de Regiones Devastadas (DGRD) tienen una lectura de carácter propagandístico, dado que las nuevas proyecciones no sólo pretendían el asentamiento, sino la creación -acorde con las pretensiones del Régimen- de todo un lenguaje genuino y representante del "nuevo orden". Como es de suponer, para dicho fin, las distintas tipologías arquitectónicas -compuestas por la vivienda rural, la iglesia, el ayuntamiento y la casa cuartel de la guardia civil- y los trazados urbanos cobran especial importancia.

Es en 1939 cuando se creará también el Instituto Nacional de Vivienda (INV), dependiente en aquel momento del Ministerio de Acción y Organización Sindical. Será en 1977 suprimido y sus funciones serán absorbidas por el Ministerio de Vivienda.

\subsection{Tipologías arquitectónicas y trazados urbanos realizados por regiones de- vastadas}

Antes de realizar un análisis de cada tipología arquitectónica y de los trazados urbanos de la DGRD, es necesario establecer una serie de cuestiones previas que complementan la comprensión acerca de cómo fue entendida la reconstrucción a manos de la Dirección.

- La primera cuestión es acerca del modo de actuación del organismo que nos ayuda a focalizar nuestra propia investigación en determinadas proyecciones. Tomando como referencia la clasificación realizada por Blanco Lage ${ }^{3}$ en Arquitectura de Regiones Devastadas, las intervenciones de los proyectos pueden diferenciarse por la incidencia de la obra

3. Blanco Lage, M., "España Una", VV.AA., Arquitectura en Regiones Devastadas. Madrid 1987, pp. 17-20. 
realizada según el grado de destrucción que la población hubiera sufrido durante la guerra civil. De este modo, se aprecian intervenciones que fueron "parciales" u otras denominadas de "nueva ordenación", o lo que es lo mismo, de nueva planta (p. 17). Las construcciones parciales se caracterizaban por tener que añadir o modificar un grupo de viviendas o algún elemento considerado como esencial para toda población, como sería una iglesia, un ayuntamiento o la Casa Cuartel de la Guardia Civil ${ }^{4}$.

- En este orden, la promoción estatal y normalizada de casas-cuartel tiene su mayor esplendor durante las décadas franquistas ${ }^{5}$, siendo un ejemplo estereotipado de las "Viviendas de Renta Limitada". Recordemos que aunque la Guardia Civil se constituyó como cuerpo en 18446, tuvo un gran protagonismo durante la Dictadura Franquista, momento en que se confirma su continuidad como fuerza de orden público, unificándose con el Cuerpo de Carabineros de Costas y Fronteras (fundado el 9 de marzo de 1829), y encomendándoseles, además de las que poseía, las misiones de vigilancia de costas y fronteras y la represión del fraude y el contrabando. $^{7}$

- La casa cuartel era el acuartelamiento característico de la Guardia Civil. Ya en su Decreto fundacional, de 28 de marzo de 1844, se recoge la necesidad de generar un cuartel donde alojar a los guardias civiles con sus familias ${ }^{8}$. Una casa cuartel era una instalación militar donde el personal allí destinado desarrollaba tanto su labor profesional como su vida privada, normalmente junto a sus familias. En España, la Guardia Civil trabajó y vivió durante muchos años en este tipo de instalaciones a las que popularmente se denominaba cuartelillos, sobre todo cuando eran de pequeñas dimensiones por encontrarse situadas en localidades de baja población y apartadas.

- El acuartelamiento tipo de la Guardia Civil se formaliza en un complejo híbrido militar-residencial, en donde la presencia de las vi-

4. Ruesga Ortuño, C., Arquitectura, estética e ideología en Reconstrucción: La obra de la Dirección General de Regiones Devastadas (1939-1953). Barcelona 2016.

5. Pinzón Ayala, D., "Las casas-cuartel de la Guardia Civil durante la II República y el franquismo: la desconocida labor de un grupo de arquitectos". Boletín Académico: Revista de investigación y arquitectura contemporánea 4 (2014), pp. 71-82.

6. Fue creada por Real Decreto de 28 de marzo de 1844 por la que se crea un cuerpo especial de fuerza armada de infantería y caballería, bajo la dependencia del Ministerio de la Gobernación de la Península y con la denominación de Guardias Civiles. Reformada el 13 de mayo de 1844.

7. Ley de 15 de marzo de 1940.

8. Real Decreto de 28 de marzo de 1844. 
viendas o pabellones adquiere un protagonismo destacado. Esto le lleva a establecer importantes paralelismos normativos, urbanísticos, formales y de obsolescencia con la realidad habitacional de periferia de muchas localidades. La ornamentación en estas Casas, estará caracterizada por "elementos decorativos "de carácter regional" que sirvan en cierto modo de telón de fondo identificativo de la arquitectura de la comarcal en la que se está trabajando". En cuanto a la forma y el aspecto de las casas cuartel, se encuentran determinadas por la conjunción de funciones que debía cumplir dentro de los poblados rurales: "defensa, vivienda, administración y acuartelamiento eventual". Para la tipología proyectada por la DGRD, en general, se juega con una serie de volúmenes en planta a partir de formas simples y fácilmente reconocibles. En planta, en la mayoría de los casos, se compone de un recinto cerrado con patio central adecuado para su función de defensa, prototipos que ya se observan en la arquitectura defensiva de castillos medievales y en los propios edificios conventuales. Para la fachada, se sigue con el mismo criterio de combinar elementos sencillos, y estará organizada por un esquema repetitivo basado en la conjunción de tres módulos (A-B-A), de modo que normalmente dos torreones sobresalgan de una parte central compuesta por menor altura9 ${ }^{9}$.

\section{El Archivo del Servicio Territorial de Badajoz}

\subsection{Origen, funciones y secciones}

El Archivo del Servicio Territorial de Badajoz, perteneciente a la actual Consejería de Movilidad, Transporte y Vivienda de la Junta de Extremadu$\mathrm{ra}^{10}$, comienza su andadura en Abril de 2010, durante la VII Legislatura (2007-2011). Forma parte del Sistema Archivístico de Extremadura. Este archivo, cumple las funciones de "archivo de gestión", de acuerdo con la Ley 2/2007 de 12 de abril, de Archivos y Patrimonio Documental de Extremadura, que manifiesta en su artículo 31, que los archivos de gestión u oficina, son:

Aquellos que están constituidos por los depósitos de documentación de uso habitual existente en las distintas oficinas de las consejerías, organismos públicos, empresas e instituciones de la Junta de Extremadura,

9. López Gómez, J. M., Un modelo de arquitectura y urbanismo franquista en Aragón: la Dirección General de Regiones Devastadas, 1939-1957. Zaragoza 1995.

10. Denominación de la Consejería en la X Legislatura (2019-2023). 
que serán responsables de dicha documentación hasta su transferencia al correspondiente Archivo Central.

Así pues, y aunque la principal función del Archivo es dar tratamiento y servicio de préstamo y consulta a la documentación de uso habitual, existe en el archivo un importante fondo histórico relativo a los proyectos de construcción Ilevados a cabo durante la Dictadura Franquista (1939-1975) y el inicio de la Democracia, que cabe destacar que fueron transferidos al Archivo del Servicio Territorial tras el traspaso de competencias estatales en materia de vivienda a la Comunidad Autónoma de Extremadura en el año $1984^{11}$.

Se han realizado para la presente investigación, tal y como ya ha quedado expuesto en la metodología, algunos análisis que ahora no reflejamos, pero que son imprescindibles para lo que más abajo exponemos:

- Por un lado, la comprensión de la estructura orgánica y funcional de las instituciones vinculadas en la generación documental;

- Y, por otro, el estudio de la génesis documental, la posterior identificación de las series documentales y la formulación de una clasificación orgánica-funcional, que ha quedado plasmado en un cuadro de clasificación, que resulta ser un instrumento descriptivo del fondo documental.

A continuación, reflejamos Sección del Cuadro de Clasificación del archivo, relativa a los proyectos de construcción generados durante el período Franquista:

01.3. SECCIÓN: ARQUITECTURA

\subsubsection{SUBSECCIÓN: PROYECTOS Y OBRAS}

01.3.1.4. Serie: Obra Sindical del Hogar (OHS) (1940-1970). Esta serie contiene la documentación relativa a la Obra Sindical del Hogar $(\mathrm{OSH})$, organismo existente durante la Dictadura Franquista. Actuaba en colaboración del Instituto Nacional de la Vivienda, su objetivo fue la construcción y administración de viviendas de construcción pública.

01.3.1.5. Serie: Obra "Educación y Descanso" (1940-1970). Esta serie contiene la documentación relativa a Educación y Descanso (EyD), organización española de tipo cultural y recreativo, existente durante la

11. Real Decreto 949/1984, de 28 de marzo, sobre traspaso de funciones y servicios del Estado a la Comunidad Autónoma de Extremadura en materia de Patrimonio Arquitectónico, Control de la Calidad de la Edificación y Vivienda (B.O.E. 21-05-1984). 
Dictadura Franquista, dedicada a la construcción de instalaciones dedicadas al ocio y el esparcimiento de los trabajadores.

01.3.1.6. Serie: Proyectos de Obra "Casas Cuartel" (1943-1971). Esta serie, contiene los proyectos de casas cuartel de la Guardia Civil, que representan la arquitectura de las instalaciones donde la Guardia Civil llevaba a cabo su labor profesional y su modo de vida.

01.3.1.7. Serie: Regiones Devastadas y Reparaciones. Esta serie, contiene los proyectos Ilevados a cabo por el Servicio Nacional de Regiones Devastadas y Reparaciones (SNRDR) (1938-1956) con la finalidad de dirigir e inspeccionar los proyectos de reconstrucción, tanto de viviendas, monumentos artísticos, como de infraestructuras, dañadas por la Guerra Civil en el territorio denominado "nacional".

01.3.1.8. Serie: Unidades Vecinales de Absorción. Esta serie contiene los proyectos de las Unidades Vecinales de Absorción (UVA). Estos proyectos, nacieron con el objetivo de realojar de forma temporal a todas las familias y personas que vivían en viviendas que no reunían unas condiciones mínimas para su habitabilidad.

01.3.1.9. Serie: Obra Sindical de Colonización. En esta serie, se encuentra la documentación relativa a la construcción de poblados llevados a cabo por el Instituto Nacional de Colonización.

01.3.1.10 Serie: Construcción de Unidades EGB. Esta serie, contiene la documentación relativa a los proyectos de construcción de colegios.

En la presente investigación nos centraremos en el análisis y estudio de la serie documental concerniente a los proyectos de construcción de casas cuartel que se Ilevaron a cabo en Extremadura entre los años 1943 y 1971.

4.2. Los expedientes de construcción de las casas cuartel en el Archivo del Servicio Territorial de Badajoz

Los expedientes que obran en el Archivo del Servicio Territorial de Badajoz pertenecientes a la Serie Documental "Proyectos de Obra de Casas Cuartel" se muestran a continuación en la siguiente tabla y figura (Tabla 2, Figura 1). En ella pueden comprobarse las localidades y los años en los que se llevaron a cabo dichos proyectos, así como los arquitectos encargados de ejecutarlos: 
Tabla 2. Proyectos de construcción de casas cuartel y arquitectos.

\begin{tabular}{|c|c|}
\hline PROYECTO & ARQUITECTO \\
\hline $\begin{array}{l}\text { Proyecto de construcción de la Casa Cuartel de } \\
\text { Salvaleón (1943) }\end{array}$ & E. García-Ormaechea Casanovas \\
\hline $\begin{array}{l}\text { Proyecto de construcción de la Casa Cuartel de Valverde } \\
\text { de Mérida (1955) }\end{array}$ & E. García-Ormaechea Casanovas \\
\hline $\begin{array}{l}\text { Proyecto de construcción de la Casa Cuartel de } \\
\text { Valdetorres (1955) }\end{array}$ & E. García-Ormaechea Casanovas \\
\hline $\begin{array}{l}\text { Proyecto de construcción de la Casa Cuartel de } \\
\text { Hornachos (1955) }\end{array}$ & E. García-Ormaechea Casanovas \\
\hline $\begin{array}{l}\text { Proyecto de construcción de la Casa Cuartel de Alconera } \\
(1955)\end{array}$ & E. García-Ormaechea Casanovas \\
\hline $\begin{array}{l}\text { Proyecto de construcción de la Casa Cuartel de } \\
\text { Cordobilla de Lácara (1956) }\end{array}$ & E. García-Ormaechea Casanovas \\
\hline $\begin{array}{l}\text { Proyecto de construcción de la Casa Cuartel de San } \\
\text { Pedro de Mérida (1956) }\end{array}$ & E. García-Ormaechea Casanovas \\
\hline $\begin{array}{l}\text { Proyecto de construcción de la Casa Cuartel de Oliva de } \\
\text { la Frontera (1957) }\end{array}$ & E. García-Ormaechea Casanovas \\
\hline $\begin{array}{l}\text { Proyecto de construcción de la Casa Cuartel de Aljucén } \\
\text { (1957) }\end{array}$ & E. García-Ormaechea Casanovas \\
\hline $\begin{array}{l}\text { Proyecto de construcción de la Casa Cuartel de Nava de } \\
\text { Santiago (1960) }\end{array}$ & E. García-Ormaechea Casanovas \\
\hline $\begin{array}{l}\text { Proyecto de construcción de la Casa Cuartel de } \\
\text { Navalvillar de Pela (1962) }\end{array}$ & E. García-Ormaechea Casanovas \\
\hline $\begin{array}{l}\text { Proyecto de construcción de la Casa Cuartel de Montijo } \\
(1962)\end{array}$ & E. García-Ormaechea Casanovas \\
\hline $\begin{array}{l}\text { Proyecto de construcción de la Casa Cuartel de Oliva de } \\
\text { la Frontera (1965) }\end{array}$ & E. García-Ormaechea Casanovas \\
\hline $\begin{array}{l}\text { Proyecto de construcción de la Casa Cuartel de } \\
\text { Burguillos del Cerro (1965) }\end{array}$ & E. García-Ormaechea Casanovas \\
\hline $\begin{array}{l}\text { Proyecto de construcción de la Casa Cuartel de Valencia } \\
\text { del Ventoso (1965) }\end{array}$ & E. García-Ormaechea Casanovas \\
\hline $\begin{array}{l}\text { Proyecto de construcción de la Casa Cuartel de Retamal } \\
\text { de Llerena (1965) }\end{array}$ & E. García-Ormaechea Casanovas \\
\hline $\begin{array}{l}\text { Proyecto de construcción de la Casa Cuartel de } \\
\text { Campanario (1966) }\end{array}$ & E. García-Ormaechea Casanovas \\
\hline $\begin{array}{l}\text { Proyecto de construcción de la Casa Cuartel de La Parra } \\
(1968)\end{array}$ & E. García-Ormaechea Casanovas \\
\hline $\begin{array}{l}\text { Proyecto de construcción de la Casa Cuartel de Helechal } \\
(1969)\end{array}$ & T. Domínguez Alonso \\
\hline $\begin{array}{l}\text { Proyecto de construcción de la Casa Cuartel de } \\
\text { Peñalsordo (1970) }\end{array}$ & T. Domínguez Alonso \\
\hline $\begin{array}{l}\text { Proyecto de construcción de la Casa Cuartel de Cheles } \\
(1971)\end{array}$ & T. Domínguez Alonso \\
\hline
\end{tabular}




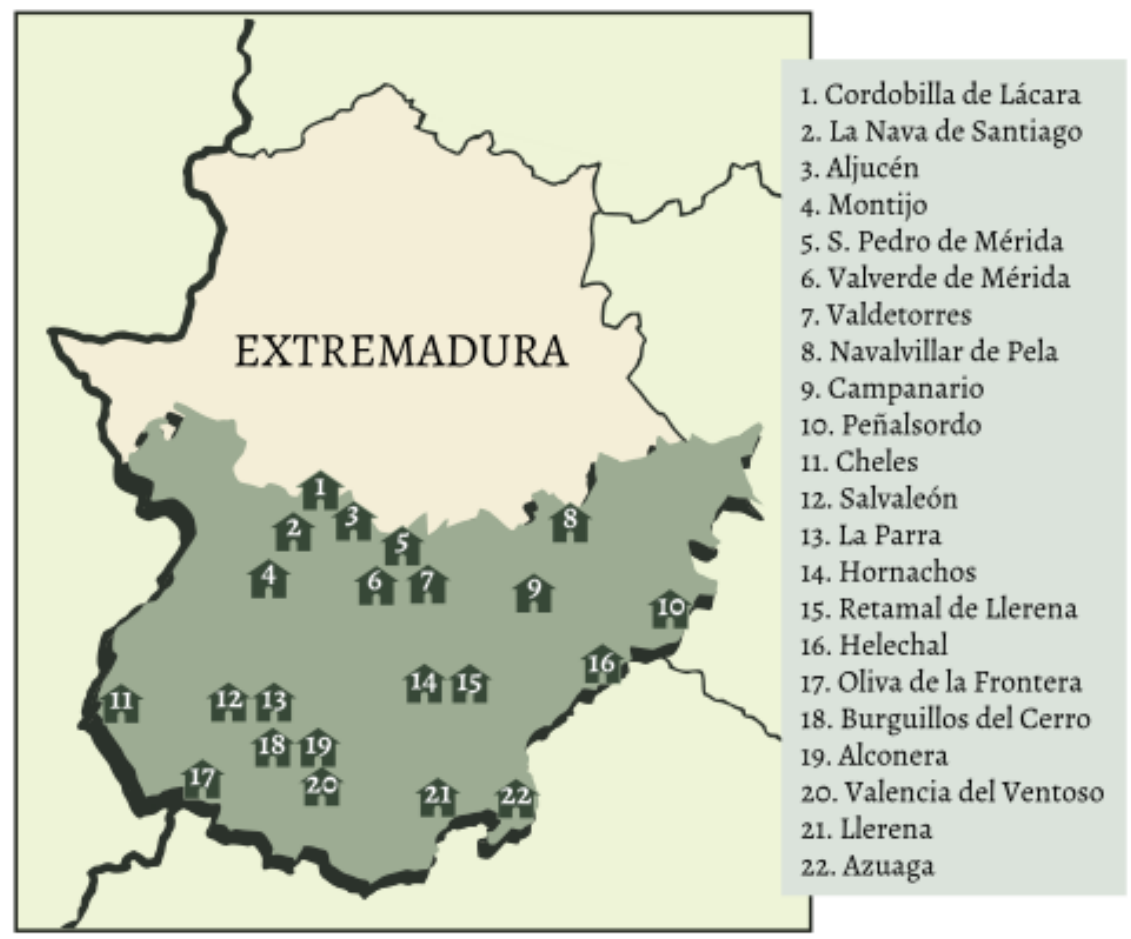

Figura 1. Proyectos de Obra de Casas Cuartel (1943-1971). Fuente: elaboración propia.

Tal y como observamos en la Tabla 2, podemos comprobar que los primeros proyectos, fueron realizados por el arquitecto Enrique García-Ormaechea Casanovas $^{12}$, funcionario del Ministerio de la Gobernación.

A partir del año 1969, los proyectos los realizará el arquitecto T. Domínguez Alonso.

Estos expedientes se caracterizan por poseer la siguiente estructura:

- Memoria.

12. Este arquitecto fue el que tuvo la relación más prolongada con la Guardia Civil. El recorrido proyectual de este arquitecto ejemplifica la evolución que experimentó la casa-cuartel estatal en el pasado siglo veinte, pues proyectó en los tres últimos periodos históricos: república, dictadura y actual democracia. El hecho de permanecer ligado a Andalucía y Badajoz posibilitó que llevara a cabo una gran cantidad de proyectos (Pinzón Ayala, D., "Las casascuartel de la Guardia Civil..., p. 77). 
- Pliego de condiciones generales para las obras acogidas al régimen de "viviendas de renta limitada".

- Capítulo I. Descripción de las obras

Artículo $1^{\circ}$. La descripción de las obras, se halla contenida en la memoria, planos y presupuesto del proyecto en cuyos documentos figuran los datos referentes a emplazamiento, disposición general, estructura, clase, cantidad y calidad de los distintos elementos y partes de la obra.

El proyecto está definido por el conjunto de todos sus elementos y no por cualquiera de estos por separado. Si apareciese alguna duda, omisión o contradicción entre los elementos del proyecto, se estará a lo que decida la Jefatura de Obras.

Artículo $2^{\circ}$. Además de las obras a que se hace referencia el artículo anterior, el Contratista estará obligado a ejecutar todas las auxiliares correspondientes que sean de la especialidad de la obra que se contrata y también las imprevistas con arreglo a los precios estipulados.

- Capítulo II. Condiciones que deben satisfacer los materiales, y la mano de obra y normas que deben regir para la ejecución de las obras.

- Capítulo III. Medición, valoración y abono de las obras.

- Capítulo IV. Obligaciones del contratista

- Capítulo V. Disposiciones generales.

- Pliego de condiciones económicas y jurídicas que habrán de regir en el contrato.

- Mediciones.

- Descomposición de precios.

- Presupuesto.

- Planos.

\section{El caso de la Casa Cuartel de Salvaleón y de la Casa Cuartel de Cheles}

A modo de ejemplo, se han seleccionado dos de los expedientes citados correspondientes a las Casas Cuartel de Salvaleón y Cheles. Ello se debe fundamentalmente a razones formales y no de contenido, esto es, por su utilidad para servir de guía o modelo identificativo de la documentación reseñada.

\subsection{El ejemplo de la "Casa Cuartel de Salvaleón"}

Se estructura de la siguiente forma:

- Memoria.

- Estudio económico. 
- Mediciones y Presupuesto.

- Descomposición de precios.

- Características resumidas.

- Planos.

- Pliego de condiciones: facultativas, económicas y jurídicas.

- La memoria del Proyecto de Construcción de la Casa Cuartel de Salvaleón, se inicia con alusiones a la victoria del bando sublevado: "El presente Proyecto tiene por objeto la terminación de la Casa Cuartel de Salvaleón, cuya construcción quedó íntimamente interrumpida al sobrevenir el Glorioso Movimiento Nacional", por tanto, podemos decir que este proyecto tiene la particularidad de ser un proyecto de continuación/terminación de un proyecto ya iniciado durante la Segunda República.

En esta memoria se describen los cuerpos y las plantas de la que constará la Casa Cuartel. Aquellos dedicados a la vida cotidiana de los Guardias dormitorios, aseos, garaje y aquellos dedicados a la vida profesional como garitas y sala de armas.

- En cuanto al estudio económico, se recogen en él los siguientes puntos:

- Valor total del cuartel.

- Reparto del valor total del cuartel.

- Operación a concertar con el INV.

- Reparto del valor de la obra ejecutada.

- Aportación de la Dirección General de la Guardia Civil a efectos de la operación con el INV.

- Aportación actual de la Dirección General de la Guardia Civil.

- Aportación del INV.

- Liquidación de la Dirección General de la Guardia Civil.

- Con respecto al capítulo de mediciones y presupuesto, en él aparecen descritos los siguientes puntos:

- Designación de Obra: edificio principal (solera, forjados, tabiques, enfoscados, rodapiés, etc.) y tapias y garitas.

- Número de unidades.

- Dimensiones.

- Resultados.

- Precio de la unidad.

- Importe (en pesetas).

- En el apartado de descomposición de precios, aparecen desglosados los precios de los materiales y los jornales (en pesetas): 
- Precios de los materiales: cemento, piedra para mampostería, arena, cal viva grasa, yeso común, ladrillos corrientes, rasillas corrientes, ladrillos huecos sencillos, baldosas hidráulicas y azulejos.

- Jornales: seguro, subsidio familiar, subsidio vejez, cuota sindical, descanso anual, fiestas no recuperables, pago de domingos.

- En el capítulo de "características resumidas" se refleja el número de viviendas que se van a construir (8 viviendas) y el nombre del arquitecto que llevará a cabo la obra, así como el tipo de viviendas y el presupuesto final de la obra:

- Tipo A: 7 viviendas con comedor, cocina, aseo y 3 dormitorios.

- Tipo B: 1 vivienda para Guardias solteros.

- Los planos que aparecen en este proyecto, son los relativos a:

- Planta baja.

- Planta principal.

- Vivienda tipo (Figura 2).

- Edificio anejo. Fachada patio (Figura 3).

\section{Coura Cuowtel de la Suazolia Cirit \\ Bivienda tifo.}
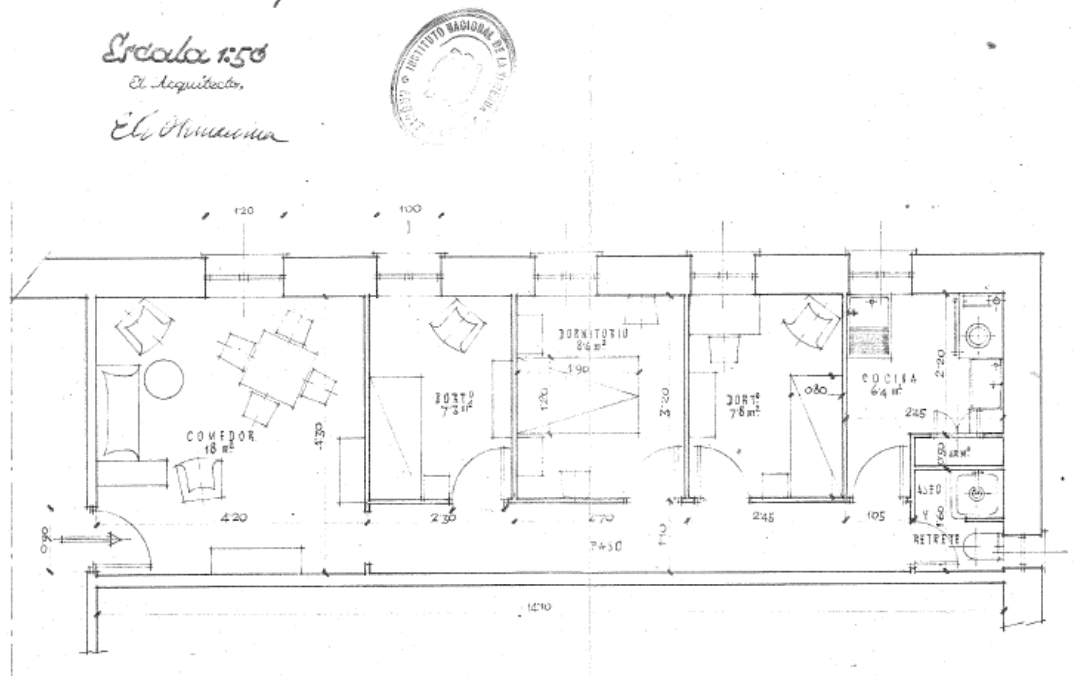

Figura 2. Plano escala 1:50 vivienda tipo "Casa Cuartel de Salvaleón". Fuente: Archivo Servicio Territorial de Badajoz. 
Cuartel de la Suardia Cifil.

Ercala 1:106.

Aalyaleór (Badajozo).

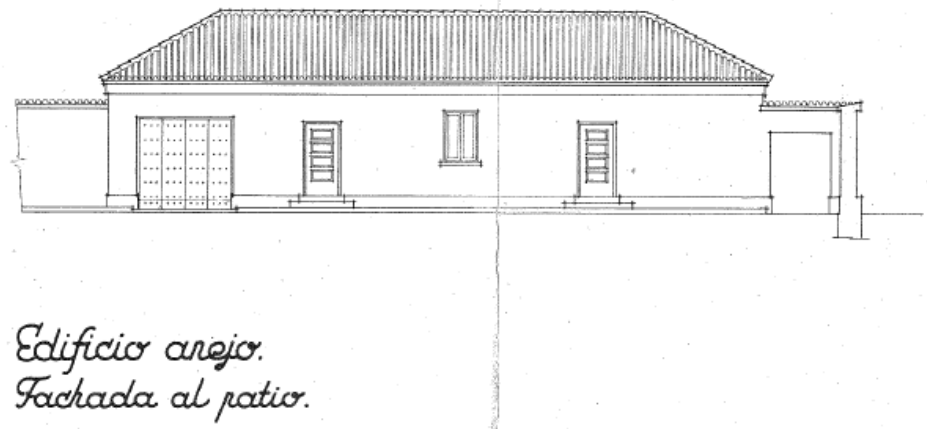

Figura 3. Plano escala 1:100 edificio anejo y fachada al patio "Casa Cuartel de Salvaleón". Fuente: Archivo Servicio Territorial de Badajoz.

En cuanto al pliego de condiciones que aparece en el proyecto, se citan las referentes a las condiciones facultativas, económicas y jurídicas, además de las generales aprobadas por Decreto de 4 de septiembre de 1908. Continuando con el ejemplo consignado, se especifican las siguientes condiciones por capítulos:

- Capítulo I. Descripción de obras.

- Capítulo II. Condiciones que deben satisfacer los materiales.

- Capítulo III. Ejecución de obras.

- Capítulo IV. Mediciones y valoración.

- Capítulo V. Liquidación y abono de las obras.

- Capítulo VI. Obligaciones del contratista.

- Capítulo VII. Disposiciones generales.

\subsection{El ejemplo de la "Casa Cuartel de Cheles"}

Los capítulos que estructuran el expediente son los siguientes:

- Memoria descriptiva. 
- Cálculo y estudio económico.

- Pliego de condiciones técnicas y legales, que recoge las órdenes de ejecución, muestras de materiales e instalación eléctrica.

- Mediciones, que incluye lo siguiente:

- Capítulo I. Movimiento de tierras.

- Capítulo II. Saneamiento.

- Capítulo III. Hormigones.

- Capítulo V. Revestimientos, solados y alicatados.

- Capítulo VI. Fontanería, fumistería y vidriería.

- Capítulo VII. Electricidad.

- Capítulo VIII. Carpintería, cerrajería y hierro laminado.

- Capítulo IX. Pintura.

- Capítulo X. Varios. En este capítulo, destacan el rótulo "Casa-Cuartel de la Guardia Civil" y el lema "Todo por la patria".

- "Descomposición de precios", donde se refleja el cuadro de precios utilitarios y el cuadro de precios auxiliares.

- Presupuesto general del proyecto.

- Y planos del proyecto. Incluye:

- 0-Plano de situación

- 1-Cimentación y saneamiento

- 2-Planta baja

- 3-Planta primera

- 4-Planta segunda

- 5-Cubiertas

- 6-Estructura

- 7-Alzado principal y lateral

- 8-Alzado posterior y lateral

- 9-Secciones A-A y B-B

- 10-Sección muro

- 11-Garaje 


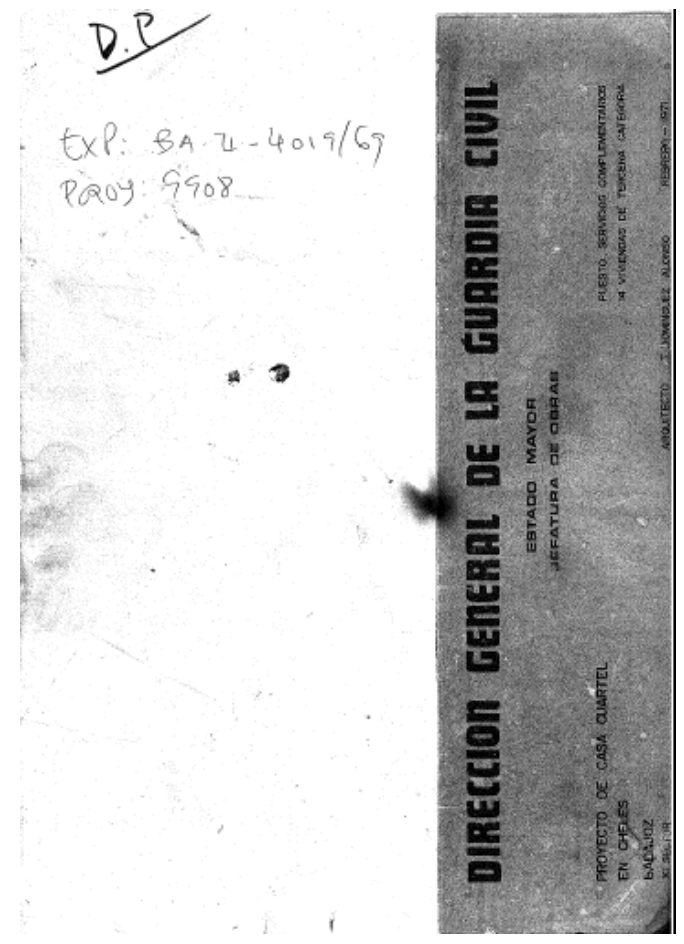

Figura 4. Cubierta del Proyecto de "Casa Cuartel de Cheles". Fuente: Archivo Servicio Territorial de Badajoz.

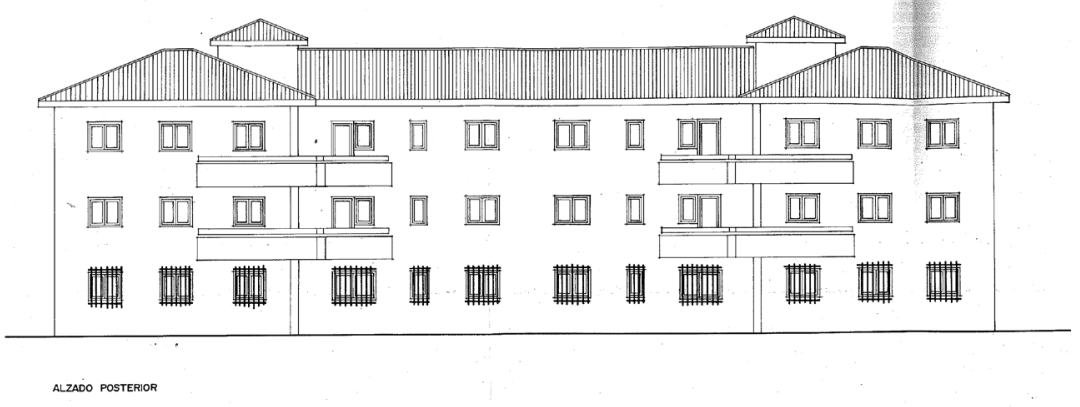

Figura 5. Plano Alzado posterior del Proyecto de "Casa Cuartel de Cheles". Fuente: Archivo Servicio Territorial de Badajoz. 


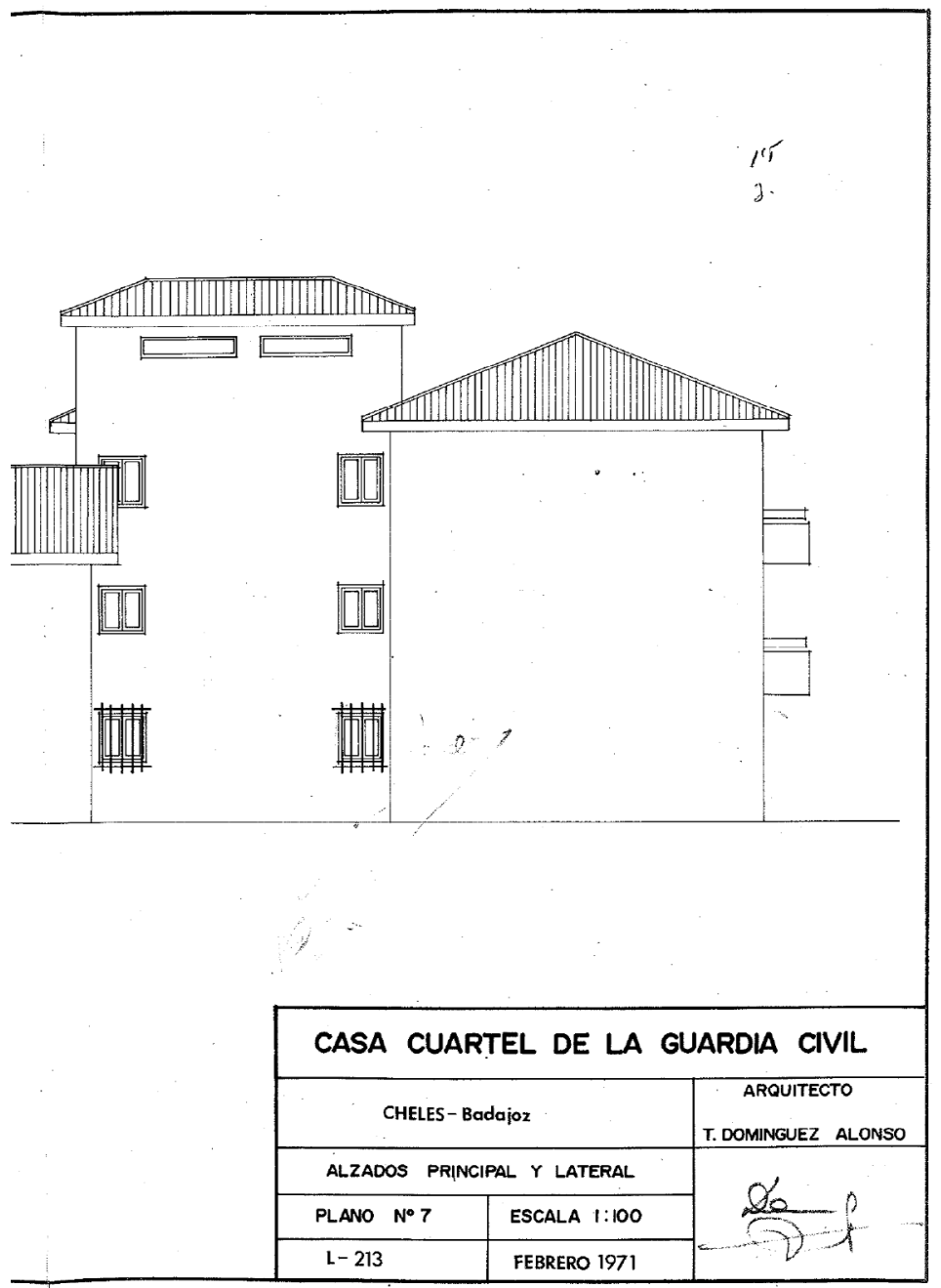

Figura 6. Plano Alzado principal y lateral del Proyecto de "Casa Cuartel de Cheles". Fuente: Archivo Servicio Territorial de Badajoz.

En cuanto a los planos, en el proyecto de la "Casa Cuartel de Salvaleón", tan sólo aparecen planos de plantas y vivienda tipo, además en algunos de los proyectos de primera época se aprovechan planos de otras Casas Cuartel de la misma región realizados con anterioridad.

Con respecto a los materiales de construcción, se continúan utilizando los mismos materiales: ladrillo, cemento, piedra, arena, cal, yeso, azulejos, etc. aunque por ejemplo dejan de utilizarse otros materiales como es el caso de 
las baldosas hidráulicas ${ }^{13}$, material muy resistente que veía utilizándose en los proyectos de primera época tanto para el interior de las viviendas como para exteriores.

\section{Importancia para la investigación histórica}

La serie "Proyectos de Obra de casas cuartel" identificada, tal y como ha quedado vislumbrado en los renglones anteriores, representa una fuente de enorme interés para la investigación histórica. Naturalmente, en este apartado únicamente mencionaremos aquellos contenidos que son factibles de ser investigados, siendo conocedores de que cada uno de ellos merecería un estudio exhaustivo.

En este orden, son varios los contenidos históricos factibles de ser analizados:

- Por un lado, contamos con los contenidos históricos que hemos denominado "naturales" o específicos de las series identificadas. Son lógicamente los contenidos de mayor interés para los investigadores dado que representan contenidos intensivos sobre determinados aspectos históricos:

- Naturalmente los aspectos relacionados con la presencia de las casas cuartel en la distribución territorial de Extremadura puede ser estudiada de forma específica con la documentación localizada. Asuntos como los motivos de su disposición geográfica, la estructura de los edificios basándose en sus diferentes funcionalidades o los precios y valores de los materiales y edificios pueden ser examinados con cierta exactitud.

- Al mismo tiempo, la historia y tipologías de las edificaciones de las casas cuartel: materias como la infraestructura de las construcciones, la disposición de los espacios en los hogares y su evolución -con lo que ello representa para la historia de la vida cotidiana-, las técnicas de construcción o la morfología de las diferentes construcciones pueden ser analizados con cierta intensidad.

- La economía de la construcción: por último, entendemos que determinados contenidos vinculados a la historia económica como los precios de las construcciones, la evolución del costo de los diferentes materiales, los sueldos de los operarios y su evolución, u otros aspectos económicos vinculados resultan factibles de ser estudiados.

- Asimismo, de forma menos intensiva, mediante la documentación identificada en nuestras series documentales, pueden ser analizados de forma

13. Este tipo de material deja de utilizarse en los años 60 (Rosell, J. y Rosell, J. R. El mosaic hidràulic. Col·legi oficial d'Aparelladors i Arquitectes tècnics de Barcelona, 1985). 
tangencial otros contenidos que, sin ser específicos, pueden complementar lo extraído a partir de otras fuentes documentales:

- Aspectos legislativos: en los diferentes expedientes analizados son abundantes las referencias a aspectos jurídicos, siendo, en consecuencia, de utilidad para el estudio de las fuentes del derecho urbanístico o el régimen jurídico consiguiente.

- Contenidos sobre la historia de la vida privada: los espacios representan algo más que lo puramente material dado que son lugares donde se desarrolla la vida de las personas. En ese orden, su estructura resulta ser una configuración del momento, siendo, en consecuencia, nuestra documentación, de cierto interés para el estudio del desarrollo de la vida cotidiana en los hogares, la disposición de sus diferentes funcionalidades y su evolución, etc.

- Por último, esta documentación puede ser utilizada igualmente para análisis económicos más generalistas. El cobro de los sueldos o el valor de los materiales descritos en los expedientes puede ser comparado con otras vertientes de carácter económico para análisis más globales.

En definitiva, la documentación identificada y analizada resulta tener un inusitado interés histórico por su carácter transversal, fundamentalmente para el s. XX.

\section{Conclusiones}

Varias son las conclusiones de nuestra investigación:

1. Por un lado, la relevancia de los archivos que conservan documentación de carácter arquitectónico. Un ejemplo es el Archivo del Servicio Territorial de Badajoz. Más allá de lo que específicamente supone para el Sistema Archivístico Extremeño, su análisis sirve para la comprensión de los Archivos Territoriales en las Comunidades Autónomas y la importancia de la documentación que dispone.

2. La utilidad de la metodología Ilevada a cabo para el análisis: por un lado, la institucional, dando lugar a la identificación de las series documentales, la documental que ha dado como lugar la descripción de las mismas y el análisis histórico que expresa su beneficio para la investigación.

3. La importancia de la serie documental estudiada: "Proyectos de Obra de Casas Cuartel". La estructura homogénea de sus expedientes nos ayuda a cocer mejor los procedimientos administrativos seguidos para la construcción de esta tipología de edificios. Del mismo modo, se ha dado a conocer a los arquitectos Ormaechea 81943-1968) y Domínguez Alonso (1969-1971) como los encargados de las construcciones en la época franquista. 
4. La importancia para la investigación histórica de la documentación analizada: por un lado, asuntos propios de la idiosincrasia de los procedimientos administrativos de la documentación y relacionados con la presencia de las casas cuartel en la provincia de Badajoz, la historia y tipología de sus edificaciones y la evolución de la visión arquitectónica, o la economía de la construcción; y, de forma transversal o tangencial con otros contenidos relacionados con los aspectos legislativos, la historia de la vida privada o análisis económicos de carácter generalista para la Extremadura de la época.

\section{Bibliografía}

Alagón Laste, J. M. y Vázquez Astorga, M., "Escuelas de «sabor agrario» en los pueblos creados por el Instituto Nacional de Colonización en la zona de La Violada-Canal de Monegros I, Aragón". Espacio, Tiempo y Educación vol. 2, 1 (2015), pp. 281-308.

Alquézar Penón, J., "Ni un hogar sin lumbre'. Política social de la vivienda en el franquismo. La Obra Sindical de la Vivienda en Andorra y Ariño". Revista de Andorra 15 (2016), pp. 91-98.

Blanco Lage, M., "España Una", Arquitectura en Regiones Devastadas. Madrid 1987, pp. 17-20.

Blasco Torrejón, B., Política de vivienda en España: un análisis global (Tesis Doctoral). Madrid 2002.

Beltrán Abadía, R., "De aquellos barros, estos lodos. La política de vivienda en la España franquista y postfranquista". Acciones e investigaciones sociales vol. 16 (2002), pp. 25-67.

Candau Pérez, M., "Acercarse a la política de vivienda en la provincia de VaIladolid: el fondo documental de la Delegación Provincial del Ministerio de la Vivienda del Archivo Histórico Provincial de Valladolid". Investigaciones históricas: Época moderna y contemporánea 25 (2005), pp. 265-280.

Capel, H., Capitalismo y morfología urbana en España. Barcelona 1975.

Castro Campano, D., "El archivo de la Delegación Provincial de Obras Públicas y Urbanismo en el Archivo Histórico Provincial de Valladolid". Investigaciones históricas: Época moderna y contemporánea 26 (2006), pp. 307-332.

Centellas Soler, M., "Los pueblos de colonización de la administración franquista en la España rural". $P+C$ : proyecto y ciudad: revista de temas de arquitectura 1 (2010), pp. 109-126.

Chueca Goitia, F., Breve historia del urbanismo: 57. Madrid 1970.

Esteban Maluenda, A., "La vivienda social española en la década de los 50: Un paseo por los poblados dirigidos de Madrid". Cuaderno de notas 7 (1999), pp. 55-80. 
Fuente Cobos, C. de la, Guía del Archivo General del Ministerio de Obras Públicas y Urbanismo. (Fondos documentales de Obras Públicas). Madrid 1983.

Gallego Domínguez, O., "Documentación de las delegaciones provinciales del Ministerio de la Vivienda e instrumentos de investigación y trabajo". Boletín de la $A N A B A D$ vol. 28, 1 (1978), pp. 101-104.

Gutiérrez-Mozo, M. E. y Caro Gallego, C., "La arquitectura de la Obra Sindical del Hogar en la ciudad de Albacete: 1941-1981". Al-Basit: Revista de estudios albacetenses 60 (2015), pp. 123-170.

Jubert, J., "La OSH: características de la gestión de la Obra Sindical del Hogar". Cuadernos de arquitectura y urbanismo 105 (1974), pp. 36-41.

Laso Ballesteros, A., Documentos de arquitectura y urbanismo en el Archivo Histórico Provincial de Valladolid: el "alma de papel". BSAA Arte 79 (2013), pp. 247-266.

Leal, A., "Régimen administrativo de los nuevos pueblos creados por el Instituto Nacional de Colonización". Revista de Estudios Agrosociales vol. 10 (1955), pp. 89-112.

López Gómez, J. M., Un modelo de arquitectura y urbanismo franquista en Aragón: la Dirección General de Regiones Devastadas, 1939-1957. Zaragoza 1995.

López Simón, Í., "El chabolismo vertical: los movimientos migratorios y la política de vivienda franquista [1955-1975]. Huarte de San Juan. Geografía e historia 25 (2018), pp. 173-192.

Pinzón Ayala, D., "Las casas-cuartel de la Guardia Civil durante la II República y el franquismo: la desconocida labor de un grupo de arquitectos". Boletín Académico: Revista de investigación y arquitectura contemporánea 4 (2014), pp. 71-82.

Pinzón Ayala, D., "Una arquitectura para trabajar y vivir en colectividad: la casa-cuartel de la Guardia Civil" (Tesis Doctoral). Sevilla 2016.

Pinzón Ayala, D., y Loren Méndez, M., "Un ejemplo de vivienda colectiva regenerable: La casa-cuartel de la Guardia Civil". I Jornadas de Periferias Urbanas 2017, pp. 96-109.

Pérez del Hoyo, R. y Gutiérrez Mozo, M. E., "Primeras políticas de vivienda en España y su influencia en la evolución de la tipología residencial: el caso de Benalúa (1883-1956)". Revista Invi vol. 28, 78 (2013), pp. 221-255.

López Díaz, J., "Vivienda social y falange: ideario y construcciones en la década de los 40". Scripta Nova. Revista electrónica de geografía y ciencias sociales vol. 7, 146 (024) (2003), Recuperado de: http://www.ub.edu/geocrit/sn/sn146(024).htm [consultado 18/11/2019].

López Gómez, J. M., Un modelo de arquitectura y urbanismo franquista en Aragón: la Dirección General de Regiones Devastadas, 1939-1957. Zaragoza 1995. 
Rojas Juárez, J. R. y De Andrés Díaz, R. Ministerio del Interior: dos siglos de historia. Bilbao 2015.

Rosell, J. y Rosell, J. R. El mosaic hidràulic. Col·legi oficial d'Aparelladors i Arquitectes tècnics de Barcelona, 1985.

Ruesga Ortuño, C., Arquitectura, estética e ideología en Reconstrucción: La obra de la Dirección General de Regiones Devastadas (1939-1953). Barcelona 2016.

Ruiz Resa, J. D. Los derechos de los trabajadores en el franquismo. Dykinson, 2015.

Sánchez Marín, Á. L., "El instituto de Reformas Sociales: origen, evolución y funcionamiento". Revista Crítica de la Historia de las Relaciones Laborales y de la Política Social 8 (2014), p. 7.

Teran, F., de. Planeamiento urbano en la España contemporánea. Historia de un proceso imposible. Barcelona 1978. 